\title{
Multimodal Resonant Scattering for Probing Morphology, Chemistry, and Kinetics of Sustainable Materials
}

\author{
Cheng Wang \\ Advanced Light Source, Lawrence Berkeley National Laboratory \\ Cwang2@lbl.gov
}

Recent development of resonant soft x-ray scattering (RSoXS) at the Advanced Light Source (ALS) has enabled its applications to many critical research areas of materials research. ${ }^{1,2}$ Combining conventional $\mathrm{x}$-ray scattering with soft $\mathrm{x}$-ray absorption spectroscopy, RSoXS is a unique chemical sensitive structure probe that provides a novel route to unambiguously decipher the complex morphologies of mesoscale materials. Tuning x-ray photon energies to match the absorption spectrum of the different chemical components, the scattering contributions from the different components can be selectively enhanced, enabling a glimpse into these complex morphologies with unprecedented details. Applications of RSoXS have been extended to the areas of structured polymer assemblies, organic electronics, functional nano-composites, as well as liquid crystals. The overarching challenge now across various disciplines is to investigate the interfacial phenomenon of new and complex materials in their operational conditions, including batteries, catalysts, gas separations, fuel cells and water desalination, and bio-hybrid systems. In order to achieve comprehensive understanding of the inoperando process, we need multimodal research tools that provide information from different perspectives in order to discover, understand, and control the interfacial phenomena and architectures. This will require combining different in situ probes, such as x-ray scattering and electron microscopy, simultaneously in the same operating condition. We will discuss the recent development of customized instrumentation, multimodal characterization methods, comprehensive theory for the extraction of the chemical distribution and spatial arrangement at multiple length scales, as well as applications to materials for sustainable future.

Liu, F., Brady, M. A., \& Wang, C. (2016).. European Polymer Journal. 88, 555-568

$2 \mathrm{Su}$, G. M., Cordova, I. A., Brady, M. A., Prendergast, D., \& Wang, C. (2016). Polymer, 99, 782-796. 